\title{
Magnetoelectric Charge Trap Memory
}

\author{
Uwe Bauer, $^{\dagger}$ Marek Przybylski, ${ }^{\ddagger}$ Jürgen Kirschner, ${ }^{\ddagger}$ and Geoffrey S. D. Beach*, ${ }^{\dagger}$ \\ ${ }^{\dagger}$ Department of Materials Science and Engineering, Massachusetts Institute of Technology, Cambridge, Massachusetts 02139, \\ United States \\ ${ }^{\ddagger}$ Max-Planck-Institut für Mikrostrukturphysik, Weinberg 2, 06120 Halle, Germany
}

\section{Supporting Information}

ABSTRACT: It is demonstrated that a charge-trapping layer placed in proximity to a ferromagnetic metal enables efficient electrical and optical control of the metal's magnetic properties. Retention of charge trapped inside the chargetrapping layer provides nonvolatility to the magnetoelectric effect and enhances its efficiency by an order of magnitude. As such, an engineered charge-trapping layer can be used to realize the magnetoelectric equivalent to today's pervasive charge trap flash memory technology. Moreover, by supplying
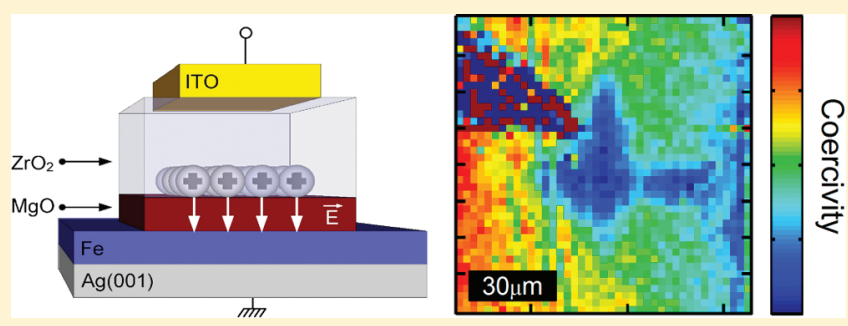

trapped charges optically instead of electrically, a focused laser beam can be used to imprint the magnetic state into a continuous metal film.

KEYWORDS: Magnetoelectric effect, electric field control of magnetic properties, charge trap flash memory, magneto-optical writing, magnetic anisotropy

$\mathrm{E}$ lectric field control of the magnetic state of a material could enable new low-power logic devices and nonvolatile memory cells. ${ }^{1-17}$ Magneto-electric coupling has typically been achieved using complex materials such as multiferroic oxides, ${ }^{5}$ dilute magnetic semiconductors, ${ }^{1,2}$ or strain-coupled magnetostrictive/piezoelectric composites. ${ }^{3}$ Only recently, direct electrical control of magnetic anisotropy, 4,6,9-12 and Curie temperature $^{8}$ has been demonstrated in transition metal ferromagnets at room temperature. These effects open the door to electrically gated spintronic devices based on materials amenable to integration with conventional semiconductor electronics.

Although magnetoelectric effects are precluded from bulk ferromagnetic metals due to the very short screening length, in films thinner than a few nanometers, spin-dependent charge screening and band level shifting can lead to pronounced electric field-driven changes to magnetic properties. ${ }^{14-17}$ Unfortunately, magnetoelectric effects in metals require a relatively strong electric field and are inherently volatile. In this work, we show that a charge-trapping layer integrated into the gate dielectric can provide the missing nonvolatility to the magnetoelectric effect and enhances its efficiency by an order of magnitude. We report the largest voltage-induced change to surface magnetic anisotropy yet demonstrated for a metallic thin film, and directly correlate this change with the density of trapped charge in an adjacent charge storage layer. This magnetoelectric charge-trap heterostructure provides a natural interface between conventional electronic and magnetic storage and logic devices, and the possibility to exploit nonvolatility in both the electronic and magnetic order offers the potential for multibit storage. ${ }^{18}$ The functionality is similar to recently proposed ferroelectric/ferromagnetic stacks ${ }^{19,20}$ but avoids the associated materials processing challenges by replacing the ferroelectric material with a well-established charge-trapping dielectric layer. Moreover, in the present implementation, charge trapping is optically assisted, providing a novel mechanism to optically manipulate the magnetic state.

We use a simple capacitor design with a heterostructure dielectric similar to that of prior studies. ${ }^{6,9,11,12}$ The bottom electrode is ferromagnetic and consists of a high quality epitaxial $\mathrm{Fe}$ film grown in wedge geometry [thickness $d_{\mathrm{Fe}}$ spanning 0-9 atomic monolayers $(\mathrm{ML})]$ on a $\mathrm{Ag}(001)$ single crystalline substrate. The bottom electrode is covered by a double-layer dielectric consisting of $10 \mathrm{~nm} \mathrm{MgO}$ and $60 \mathrm{~nm} \mathrm{ZrO}_{2} . \mathrm{MgO}$ grows epitaxially on $\mathrm{Fe}(001),{ }^{21}$ and the $\mathrm{Fe} / \mathrm{MgO}$ interface exhibits a strong interfacial magnetic anisotropy. ${ }^{16}$ Moreover, the $\mathrm{Fe} /$ $\mathrm{MgO}$ system allows for eventual integration with a magnetic tunnel junction device for magnetoresistive read out. The $\mathrm{MgO}$ layer also acts as a wide bandgap barrier between $\mathrm{Fe}$ and $\mathrm{ZrO}_{2}$, which facilitates trapped charge storage in the latter. The sputter deposited $\mathrm{ZrO}_{2}$ overlayer was chosen not only because of its high dielectric constant ${ }^{9}$ but also because of its high charge trap density which makes it a promising candidate for future charge trap memory devices. ${ }^{22}$ In order to provide optical access to the $\mathrm{Fe}$ bottom electrode, transparent conducting gate electrodes with a diameter of $70 \mu \mathrm{m}$ were made of $30 \mathrm{~nm}$ thick indium tin oxide (ITO). Magnetic properties were probed via the polar magneto-optical Kerr effect (MOKE), using a diode laser with $532 \mathrm{~nm}$ wavelength focused to a $\sim 5 \mu \mathrm{m}$ diameter

Received: November 22, 2011

Revised: January 25, 2012

Published: February 2, 2012 
a
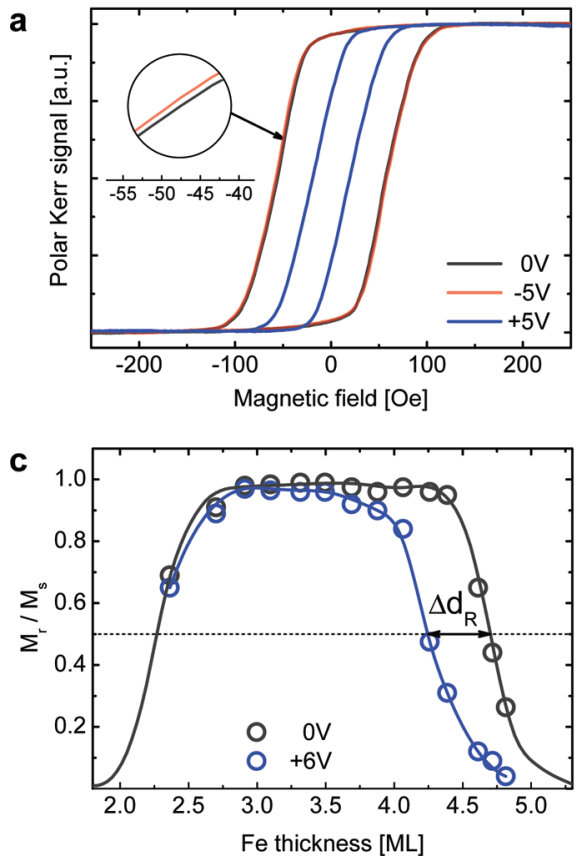

b

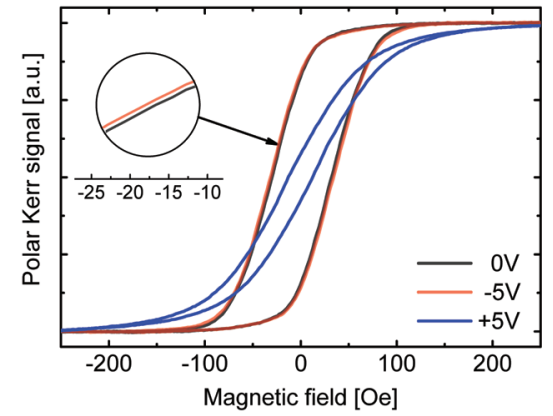

d

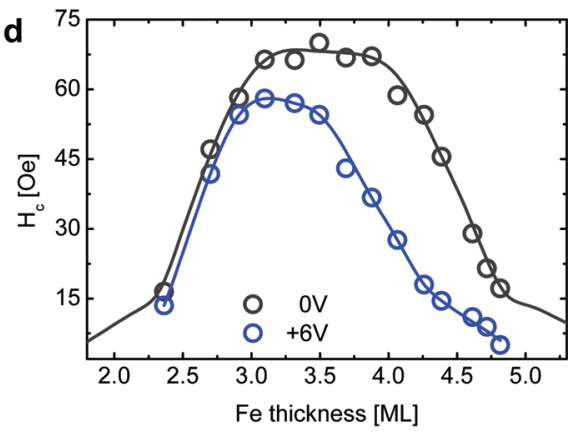

Figure 1. (a,b) Exemplary polar MOKE hysteresis loops measured under an external bias voltage of $0,-5$, and $+5 \mathrm{~V}$ at $4.3 \mathrm{ML}$ Fe (a) and $4.6 \mathrm{ML}$ Fe (b). Inset in $(\mathrm{a}, \mathrm{b})$ shows magnified section of hysteresis loops to highlight asymmetry of bias voltage effects under inversion of bias polarity. (c,d) Thickness dependence of $M_{\mathrm{r}} / M_{\mathrm{s}}(\mathrm{c})$ and $H_{\mathrm{c}}(\mathrm{d})$ on continuous Fe wedge (black line) and on discrete ITO electrodes at bias voltage of $0 \mathrm{~V}$ (black circle) and $+6 \mathrm{~V}$ (blue circle). Note, blue line in $(\mathrm{c}, \mathrm{d})$ is a guide to the eye and $\Delta d_{\mathrm{R}}$ indicates the voltage induced shift in spin reorientation thickness $(\mathrm{c})$.

probe spot and positioned using a high-resolution sample scanning stage. The coercive field $H_{c}$ and remanent to saturation magnetization ratio $M_{\mathrm{r}} / M_{\mathrm{s}}$ were derived from MOKE hysteresis loops and their modifications, $\Delta H_{c}$ and $\Delta M_{\mathrm{r}} / M_{\mathrm{s}}$, were used to characterize the influence of the external bias voltage and trapped charges on magnetic properties of the Fe bottom electrode.

We observe a giant modification of $H_{\mathrm{c}}$ and $M_{\mathrm{r}} / M_{\mathrm{s}}$ if a positive bias voltage $\left(V_{\mathrm{b}}\right)$ of just a few volts is applied to the ITO gate electrode. This modification depends strongly on $\mathrm{Fe}$ thickness and therefore on the strength of perpendicular magnetic anisotropy. Exemplary hysteresis loops, at a $\mathrm{Fe}$ thickness of 4.3 and 4.6 ML showing a strong modification of $\Delta H_{\mathrm{c}}=-37$ Oe or $-66 \%$ and of $\Delta M_{\mathrm{r}} / M_{\mathrm{s}}=-0.53$ or $-78 \%$ can be seen in Figure 1a,b, respectively. The Fe thickness dependence of $M_{\mathrm{r}} / M_{\mathrm{s}}$ and $H_{\mathrm{c}}$ at zero bias and at $V_{\mathrm{b}}=+6 \mathrm{~V}$ is shown in Figure 1c,d, respectively. From there it can be seen that up to a spin reorientation thickness of $4.7 \mathrm{ML} \mathrm{Fe}$, the magnetization vector is aligned perpendicular to the film plane and only above this thickness prefers an orientation in the film plane. The apparent loss of perpendicular anisotropy below 2.5 $\mathrm{ML} \mathrm{Fe}$ is due to the thickness dependence of the Curie temperature, which decreases below RT at this thickness. ${ }^{23}$

The orientation of the magnetization vector in ultrathin metallic ferromagnets is often dictated by a surface contribution, $K_{\mathrm{S}}$, to the magnetic anisotropy energy. ${ }^{24}$ At the spin reorientation transition, perpendicular surface magnetic anisotropy $K_{S}$ and shape anisotropy of the Fe film compensate each other:

$$
\frac{K_{\mathrm{S}}}{d_{\mathrm{R}}}=2 \pi \mathrm{M}_{\mathrm{s}}^{2}
$$

Here, $d_{\mathrm{R}}$ and $M_{\mathrm{s}}$ are the spin reorientation thickness and the saturation magnetization of the Fe film, respectively. From Figure 1c, application of $V_{\mathrm{b}}=+6 \mathrm{~V}$ shifts the spin reorientation thickness $d_{\mathrm{R}}$ downward by $\Delta d_{\mathrm{R}} \approx-0.45 \mathrm{ML}$. Taking $M_{\mathrm{s}}=1710 \mathrm{G}$ we therefore estimate the change in perpendicular surface anisotropy as $\Delta K_{\mathrm{S}}=2 \pi M_{\mathrm{S}}^{2} \Delta d_{\mathrm{R}}=118 \mu \mathrm{J} / \mathrm{m}^{2}$. This change is an order of magnitude larger than previously reported ${ }^{6}$ for $\mathrm{Fe} / \mathrm{MgO}$, despite the much lower applied voltage in the present case. Considering the thicknesses and dielectric constants of the $\mathrm{MgO} / \mathrm{ZrO}_{2}$ stack, this would correspond to a giant electric field effect on magnetic anisotropy of $944 \mathrm{fJ} / \mathrm{Vm}$, the largest magnetoelectric efficiency yet reported for any metallic system, and far beyond theoretical predictions ${ }^{16}$ of $100 \mathrm{fJ} / \mathrm{Vm}$.

In contrast to a positive bias, application of negative bias results in only a weak modification of $H_{\mathrm{c}}$ and $M_{\mathrm{r}} / M_{\mathrm{s}}$. Under a bias voltage of $-5 \mathrm{~V}$, an increase of $2 \%$ in $H_{\mathrm{c}}$ and of $3 \%$ in $M_{\mathrm{r}} / M_{\mathrm{s}}$ is observed at the same Fe thicknesses (see Figure 1a and $b$ ). These values are similar to previously reported results ${ }^{4,13}$ but more than 1 order of magnitude smaller than the changes observed here under the positive bias.

Considering the asymmetry under bias inversion, together with the giant magnitude under positive bias, it is clear that the electric field from the external bias alone cannot directly account for the observed magnetoelectric effects (see also Supporting Information). Instead, an internal electric field originating from charge trapped in the gate dielectric could play a key role. In fact it has been suggested ${ }^{10-12}$ that trapped charge could play a role in the magnetoelectric effect that may help to explain the wide range of magnetoelectric efficiencies reported in the literature. ${ }^{4,6,9-13}$

If trapped charges are indeed responsible for the observed giant modification of magnetic anisotropy, we would expect the modifications of $H_{\mathrm{c}}$ and $M_{\mathrm{r}} / M_{\mathrm{s}}$ to persist long after $V_{\mathrm{b}}$ is removed. $^{22}$ This is exactly what we find experimentally (Figure 2a,b). In Figure 2b, scanning MOKE maps showing $M_{\mathrm{r}} / M_{\mathrm{s}}$ in a $100 \times 100 \mu \mathrm{m}$ area around an ITO electrode are displayed. After measuring an initial MOKE map, a bias of $+5 \mathrm{~V}$ was applied to the ITO electrode while $M_{\mathrm{r}} / M_{\mathrm{s}}$ was monitored until the maximum reduction was attained. The bias voltage was then 


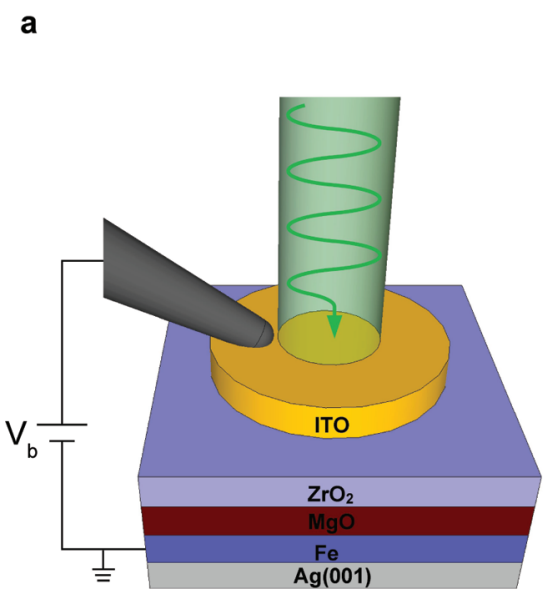

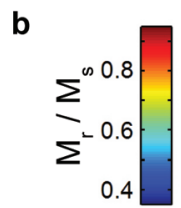
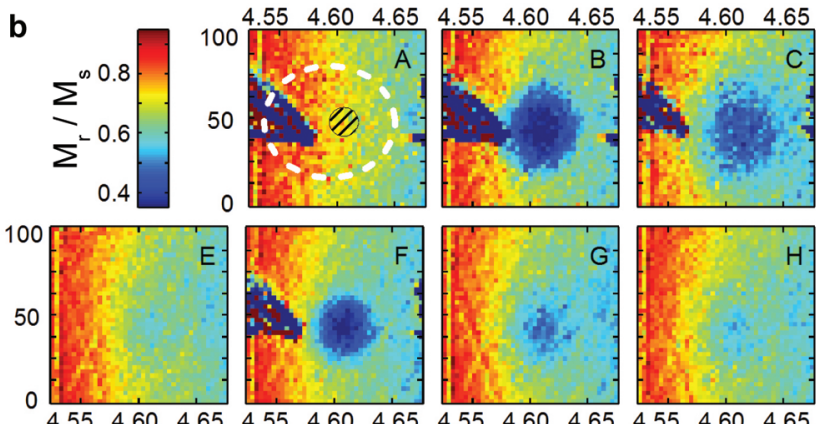$$
\text { C }
$$
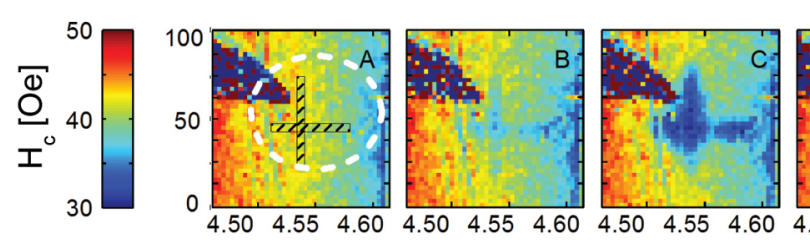

Fe thickness [ML]

Figure 2. (a) Schematics of experimental setup including probe tip to apply bias voltage $V_{\mathrm{b}}$ to the device and focused laser beam to measure scanning MOKE maps. (b) MOKE maps showing $M_{\mathrm{r}} / M_{\mathrm{s}}$ in $100 \times 100 \mu \mathrm{m}$ area around ITO electrode (a). After measuring an initial MOKE map (A), a gate voltage of $+5 \mathrm{~V}$ is applied while laser is focused on the center of ITO electrode. Dashed white line indicates circumference of electrode while dashed black area indicates position of laser beam during voltage application. $1 \mathrm{~h}$ after bias voltage is removed, a MOKE map of same area is measured (B), showing retention of modification of $M_{\mathrm{r}} / M_{\mathrm{s}}$ in area exposed to laser light during bias application. Additional MOKE maps are measured after 8 (C), $24(\mathrm{D})$, and $72 \mathrm{~h}(\mathrm{E})$, showing slow decay of $M_{\mathrm{r}} / M_{\mathrm{s}}$ back to its initial state. After $72 \mathrm{~h}$, gate voltage of $+5 \mathrm{~V}$ is applied again while the focused laser beam is placed in center of ITO pad and MOKE maps measured 1 (F), $8(\mathrm{G}), 24(\mathrm{H})$, and $72 \mathrm{~h}$ (I) after second voltage application are shown. Small changes in area showing modification in $M_{\mathrm{r}} / M_{\mathrm{s}}$ for MOKE maps taken after first and second voltage application is due to small change in focal distance and correspondingly laser beam size over time. (c) MOKE maps showing $H_{\mathrm{c}}$ in $100 \times 100 \mu \mathrm{m}$ area around ITO electrode. After measuring initial MOKE map (A), a gate voltage of $+3 \mathrm{~V}$ is applied and two perpendicular lines are rastered with focused laser beam (dashed area). After gate voltage is removed, MOKE map (B) shows local modification of $H_{c}$ in area rastered by laser beam. Re-exposure with 5 times higher exposure time results in stronger local modification of $H_{c}(\mathrm{C})$. Scanning laser beam across whole $100 \times 100 \mu \mathrm{m}$ area around ITO electrode under a bias of $+3 \mathrm{~V}$ modifies $H_{c}$ across whole gate electrode but not outside (D), illustrating that both laser illumination and gate voltage are necessary to modify $H_{c}$.

removed and MOKE maps were measured after 1, 8, 24, and $72 \mathrm{~h}$. From the images in Figure $2 \mathrm{~b}$, the modifications of $M_{\mathrm{r}} / M_{\mathrm{s}}$ remain clearly visible even $24 \mathrm{~h}$ after the positive bias was removed and only after $72 \mathrm{~h}$ do the magnetic properties return close to their initial values. This sequence was then repeated with similar results.

Notably, rather than occurring uniformly across the ITO electrode, the reduction of $M_{\mathrm{r}} / M_{\mathrm{s}}$ appears strongest near the electrode center. This is exactly the region that was illuminated by the MOKE probe laser during voltage application. To better understand the mutual dependence of external bias and laser radiation and their influence on magnetic properties, Figure $2 \mathrm{c}$ shows results after the focused laser spot was rastered along two orthogonal lines while a positive bias of $+3 \mathrm{~V}$ was applied to the ITO electrode. After $V_{\mathrm{b}}$ was removed, two orthogonal lines are clearly visible as a local reduction of $H_{c}$ imprinted within the continuous film. Upon re-exposure with a 5 -fold increase in illumination time, the imprinted magnetic pattern is increasingly well-defined. Finally, after rastering the laser across the entire $100 \times 100 \mu \mathrm{m}$ map area with $V_{\mathrm{b}}=+3 \mathrm{~V}$, a reduction of $H_{c}$ across the entire electrode area was observed, whereas regions outside the electrode area remain unaffected. This observation, as well as the observed asymmetry of $\Delta H_{c}$ and $\Delta M_{\mathrm{r}} / M_{\mathrm{s}}$ under bias inversion (Figure 1a,b) clearly show that the modifications of $H_{\mathrm{c}}$ and $M_{\mathrm{r}} / M_{\mathrm{s}}$ are of electronic origin and not the result of a simple heating effect.

Together, the results shown in Figure 2 suggest that trapped charge within the gate dielectric is responsible for the magnitude and retention characteristics of the observed magnetoelectric effects, and that in this system these charges are supplied through an optically assisted process. ${ }^{25}$ This heterostructure hence offers the unique possibility of optical definition and detection of magnetic patterns within a continuous magnetic medium. ${ }^{26}$ Under application of a positive gate bias, the laser may be used to imprint magnetic data into the continuous magnetic film, while in the absence of the gate bias the same laser can be used to read out those data nondestructively.

We have measured the device capacitance in order to directly correlate the trapped charge density with changes in magnetic anisotropy. In metal insulator metal capacitors, the presence of trapped charge manifests as a capacitance increase due to an increase in the polarizability of the insulating layer. ${ }^{27,28} \mathrm{We}$ measure an initial device capacitance of $\sim 9.7 \mathrm{pF}$ at zero bias, which is in agreement with expectations assuming dielectric constants of $\varepsilon=10$ and $\varepsilon=25$ for the $\mathrm{MgO}$ and $\mathrm{ZrO}_{2}$ layer, respectively. After applying $V_{\mathrm{b}}=+3 \mathrm{~V}$ while simultaneously illuminating the electrode for $180 \mathrm{~s}$, the capacitance increased significantly, by $\Delta C=0.73 \mathrm{pF}$ or $7.5 \%$. This capacitance increase is accompanied by a decrease of $M_{\mathrm{r}} / M_{\mathrm{s}}$ by $\Delta M_{\mathrm{r}} / M_{\mathrm{s}}=$ -0.48 . Upon removal of $V_{\mathrm{b}}$, both $\Delta C$ and $\Delta M_{\mathrm{r}} / M_{\mathrm{s}}$ decayed with time (Figure $3 \mathrm{a}$ ) at the same rate, dropping to half their initial values at $\sim 3700 \mathrm{~s}$. As seen in Figure $3 \mathrm{~b}$, application of a negative bias increases the relaxation rate of $M_{\mathrm{r}} / M_{\mathrm{s}}$ by an order of magnitude, while positive $V_{\mathrm{b}}$ leads to retention of $\Delta M_{\mathrm{r}} / M_{\mathrm{s}}$ over time.

The behavior depicted in Figure $3 b$ is reminiscent of the write, erase and retention characteristics of charge trap flash (CTF) memory devices. ${ }^{29}$ The difference here is that the shift in gate voltage $\Delta V$ is replaced by $\Delta M_{\mathrm{r}} / M_{\mathrm{s}}$. In its simplest form, a conventional CTF memory device consists of a doped 


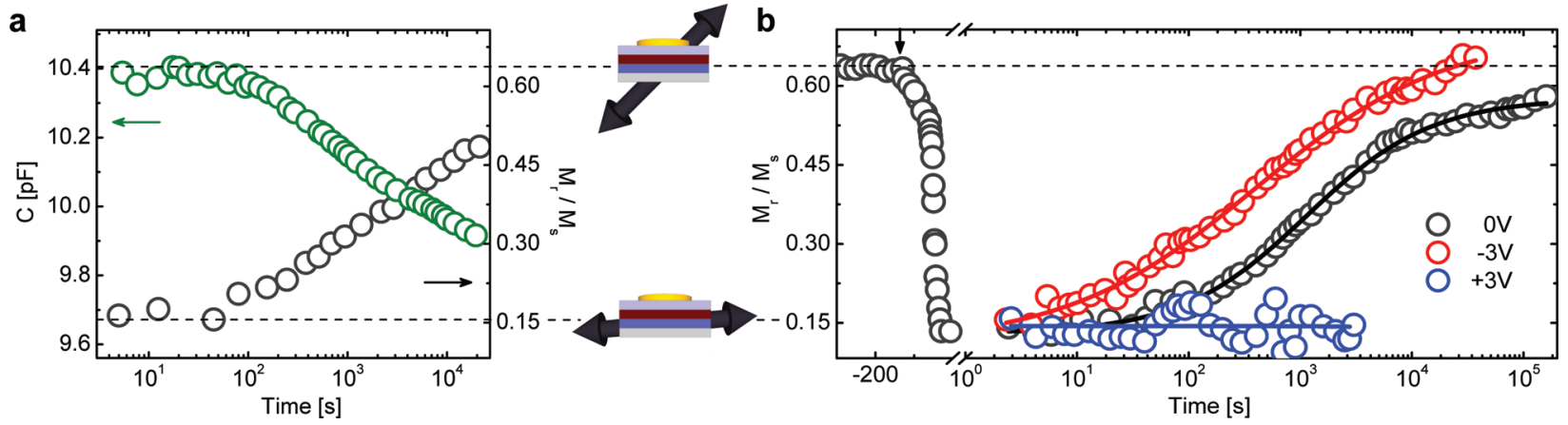

Figure 3. (a) Evolution of capacitance $C$ (green circle) and $M_{\mathrm{r}} / M_{\mathrm{s}}$ (black circle) over time after a bias voltage of $+3 \mathrm{~V}$ was applied for $180 \mathrm{~s}$ under laser illumination. (b) Time dependence of $M_{\mathrm{r}} / M_{\mathrm{s}}$ at 4.6 ML Fe under different bias conditions. Under continuous laser illumination, a positive gate voltage of $+3 \mathrm{~V}$ is applied at $t=-150 \mathrm{~s}$ until $t=0 \mathrm{~s}$ and $M_{\mathrm{r}} / M_{\mathrm{s}}$ saturates after $\sim 100 \mathrm{~s}$. After saturation the gate voltage is either kept constant $(+3 \mathrm{~V}$, blue circle), inverted $\left(-3 \mathrm{~V}\right.$, red circle) or removed $\left(0 \mathrm{~V}\right.$, black circle) and the evolution of $M_{\mathrm{r}} / M_{\mathrm{s}}$ is measured over time. For the case of $0 \mathrm{~V}$ and $-3 \mathrm{~V}$ gate voltage, $M_{\mathrm{r}} / M_{\mathrm{s}}$ is sigmoid function of time, whereas at $+3 \mathrm{~V}$ it remains constant. Device schematics in center show orientation of magnetic easy axis corresponding to indicated values of $M_{\mathrm{r}} / M_{\mathrm{s}}$.

semiconducting bottom electrode, wide band gap tunnel barrier, charge trapping layer and metallic gate electrode. ${ }^{29,30}$ The present structure is very similar in design but instead of a doped semiconductor, we use a ferromagnetic metal bottom electrode. $\mathrm{MgO}$ serves as the wide band gap barrier and $\mathrm{ZrO}_{2}$ as the charge trapping layer. In the present device we observe retention times of several days; however, addition of a blocking layer to the dielectric stack should allow for retention times in excess of 10 years, which is routinely achieved in conventional CTF memory devices. ${ }^{22}$

Our experimental results suggest that under positive gate bias, charge is optically pumped from either the top or the bottom electrode into the $\mathrm{ZrO}_{2}$ charge trapping layer. Both electron injection from the Fe electrode and hole injection from the ITO electrode (Figure 4a) would explain the observed

a

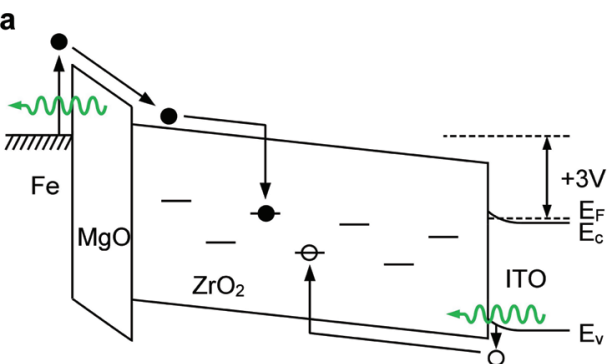

b

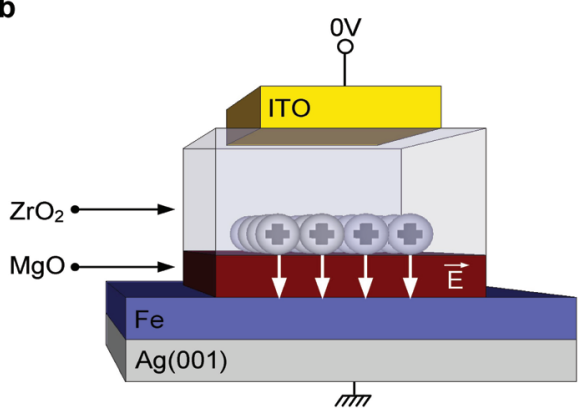

Figure 4. (a) Schematic band structure of device at $+3 \mathrm{~V}$ gate bias illustrating two possibilities of optically assisted charge injection into $\mathrm{ZrO}_{2}$ charge trapping layer. Here, $E_{\mathrm{V}}, E_{\mathrm{C}}$ and $E_{\mathrm{F}}$ correspond to the valence band, conduction band and Fermi level, respectively. (b) Under positive gate bias, holes from ITO electrode can be optically pumped into $\mathrm{ZrO}_{2}$ charge trapping layer. Trapped holes remain in $\mathrm{ZrO}_{2}$ trapping layer after external bias is removed and generate internal electric field $\vec{E}$ across $\mathrm{MgO}$ barrier. asymmetry of $\Delta H_{\mathrm{c}}$ and $\Delta M_{\mathrm{r}} / M_{\mathrm{s}}$ under inversion of bias polarity (Figure 1a,b). However, with a thickness of $10 \mathrm{~nm}$, the $\mathrm{MgO}$ layer is too thick for direct tunneling from the bottom electrode to the $\mathrm{ZrO}_{2}$ charge trapping layer, ${ }^{31}$ suggesting the ITO gate electrode acts as the source for charge injection. Additional capacitance-voltage measurements (see Supporting Information) show that dominantly positive charge is trapped in the $\mathrm{ZrO}_{2}$ layer, which indicates that the injected carriers are holes, and that optical illumination likely functions to assist hole injection across the $\mathrm{ZrO}_{2} /$ ITO valence band offset. The optically assisted nature of charge trapping together with the higher defect density expected for sputter deposited $\mathrm{ZrO}_{2}$ explains why charge trapping effects are far more prominent here than in previous magnetoelectric measurements ${ }^{9,32}$ with $\mathrm{ZrO}_{2}$ as the gate dielectric.

After gate bias and illumination are removed, holes remain in the $\mathrm{ZrO}_{2}$ layer and result in an internal electric field across the $\mathrm{MgO}$ barrier (Figure 4b), which modifies the magnetic properties of the Fe film. With a positive charge trap density of up to $\sim 2 \times 10^{13} \mathrm{~cm}^{-2}$ (see Supporting Information), we arrive at an internal electric field across the $\mathrm{MgO}$ barrier of $\sim 0.3 \mathrm{~V} / \mathrm{nm}$. This internal electric field is of same polarity but many times larger than the external electric field due to $V_{\mathrm{b}}$, which explains the observed giant modification of magnetic anisotropy. As charge slowly leaks from the trapping layer, the electric field across the $\mathrm{MgO}$ layer decreases and magnetic anisotropy simultaneously decays to its initial value. Addition of a blocking layer to the dielectric stack should significantly enhance retention times, ${ }^{22}$ permitting nonvolatile functioning of metallic magnetoelectric devices.

In summary, we have shown that trapped charges and not the external bias can play the key role in the magnetoelectric effect. Our results provide a new means to engineer magnetic properties at the nanoscale and may lead to novel functional nanodevices exploiting the coupling between nonvolatile electronic and magnetic order. Trapped charges are essential to achieve a giant modification of magnetic anisotropy and the type of trapped charges is crucial to determine the polarity of the effect. Moreover, engineering of hesterostructure devices with charge trapping layers allows the design of magnetoelectric charge trap (MECT) memory cells by adding the missing nonvolatility to the magnetoelectric effect. Engineering the band offsets or layer thicknesses of the presented device should allow for completely electrical charge injection, while charge 
injection by optical photons opens the path to novel magnetooptoelectronic hybrid devices. Recently, adjacent ferromagnetic and ferroelectric layers were proposed ${ }^{19,20}$ as a means to electrically control ferromagnetism. Our results show that a charge trapping layer placed adjacent to the ferromagnet can provide the same functionality with the advantage of using simple oxide dielectrics compatible with existing semiconductor processing technology.

Methods. $\operatorname{Ag}(001) / \mathrm{Fe} / \mathrm{MgO}$ samples were fabricated in a multichamber ultra high vacuum (UHV) system with base pressure better than $2 \times 10^{-10}$ mbar. $\mathrm{Ag}(001)$ single crystal substrates were prepared by several sputter and annealing cycles at $600{ }^{\circ} \mathrm{C}$. Fe films were epitaxially grown by molecular beam epitaxy and after deposition the Fe films were annealed in UHV for $30 \mathrm{~min}$ at $150{ }^{\circ} \mathrm{C}$ to improve surface morphology. ${ }^{23} \mathrm{MgO}$ was deposited at room temperature (RT) from a stochiometric target by electron beam evaporation at a pressure below $9 \times$ $10^{-10}$ mbar. The samples were then covered by $\mathrm{ZrO}_{2}$ films grown at RT by reactive sputter deposition from a $\mathrm{Zr}$ target under $4.0 \mathrm{scc} / \mathrm{m} \mathrm{O}_{2}$ flow. ITO electrodes were fabricated with a shadow mask and sputter deposited from a stochiometric ITO (90/10) target also at RT. Electrical contact to the ITO electrodes was made with $\mathrm{W}$ cat whisker probe tips with $1 \mu \mathrm{m}$ tip diameter. Capacitance was measured with an Agilent 4284A LCR meter at a frequency of $1 \mathrm{kHz}$ and $\mathrm{AC}$ amplitude of $25 \mathrm{mV}$. MOKE measurements were performed on a scanning MOKE system with a diode laser with $532 \mathrm{~nm}$ wavelength focused to a $\sim 5 \mu \mathrm{m}$ diameter probe spot. By using an additional polarizer as a variable attenuator, the optical power density can be varied between $2 \times 10^{3}$ to $8 \times 10^{4} \mathrm{~W} / \mathrm{cm} 2$. MOKE hysteresis loops were measured at a typical sweep rate of the magnetic field of $10 \mathrm{~Hz}$.

\section{ASSOCIATED CONTENT}

\section{S Supporting Information}

Additional figures showing asymmetric response under bias inversion, illumination effects, illumination dependence of relaxation measurements and capacitance voltage characteristics. Expanded discussion of charge trap density, charge trap induced internal electric field. and optically assisted charge trapping process. This material is available free of charge via the Internet at http://pubs.acs.org.

\section{AUTHOR INFORMATION}

\section{Corresponding Author}

*E-mail: gbeach@mit.edu. Tel: +1 617 258-0804.

\section{Notes}

The authors declare no competing financial interest.

\section{ACKNOWLEDGMENTS}

U.B. and G.B. acknowledge support under NSF-ECCS -1128439. Technical support from $H$. Menge, W. Greie, D. Bono, and M. Tarkanian is gratefully acknowledged.

\section{REFERENCES}

(1) Ohno, H.; et al. Electric-field control of ferromagnetism. Nature 2000, 408, 944-946.

(2) Chiba, D.; Yamanouchi, M.; Matsukura, F.; Ohno, H. Electrical manipulation of magnetization reversal in a ferromagnetic semiconductor. Science 2003, 301, 943-945.

(3) Eerenstein, W.; Mathur, N. D.; Scott, J. F. Multiferroic and magnetoelectric materials. Nature 2006, 442, 759-765.
(4) Weisheit, M. Electric field-induced modification of magnetism in thin-film ferromagnets. Science 2007, 315, 1077-1077.

(5) Ramesh, R; Spaldin, N. A. Multiferroics: progress and prospects in thin films. Nat. Mater. 2007, 6, 21-29.

(6) Maruyama, T.; et al. Large voltage-induced magnetic anisotropy change in a few atomic layers of iron. Nat. Nanotechnol. 2009, 4, $158-161$

(7) Gerhard, L.; et al. Magnetoelectric coupling at metal surfaces. Nat. Nanotechnol. 2010, 5, 792-797.

(8) Chiba, D.; et al. Electrical control of the ferromagnetic phase transition in cobalt at room temperature. Nat. Mater. 2011, 10, 853-856.

(9) Endo, M.; Kanai, S.; Ikeda, S.; Matsukura, F.; Ohno, H. Electricfield effects on thickness dependent magnetic anisotropy of sputtered MgO/Co40Fe40B20/Ta structures. Appl. Phys. Lett. 2010, 96, 212503.

(10) Nozaki, T.; Shiota, Y.; Shiraishi, M.; Shinjo, T.; Suzuki, Y. Voltage-induced perpendicular magnetic anisotropy change in magnetic tunnel junctions. Appl. Phys. Lett. 2010, 96, 022506.

(11) Bonell, F.; et al. Large change in perpendicular magnetic anisotropy induced by an electric field in FePd ultrathin films. Appl. Phys. Lett. 2011, 98, 232510.

(12) Ha, S. S.; et al. Voltage induced magnetic anisotropy change in ultrathin $\mathrm{Fe}(80) \mathrm{Co}(20) / \mathrm{MgO}$ junctions with Brillouin light scattering. Appl. Phys. Lett. 2010, 96, 142512.

(13) Fowley, C.; Rode, K.; Oguz, K.; Kurt, H.; Coey, J. M. D. Electric field induced changes in the coercivity of a thin-film ferromagnet. J. Phys. D: Appl. Phys. 2011, 44, 305001.

(14) Duan, C. G.; et al. Surface magnetoelectric effect in ferromagnetic metal films. Phys. Rev. Lett. 2008, 101, 137201.

(15) Nakamura, K.; et al. Giant modification of the magnetocrystalline anisotropy in transition-metal monolayers by an external electric field. Phys. Rev. Lett. 2009, 102, 187201.

(16) Niranjan, M. K.; Duan, C. G.; Jaswal, S. S.; Tsymbal, E. Y. Electric field effect on magnetization at the $\mathrm{Fe} / \mathrm{MgO}(001)$ interface. Appl. Phys. Lett. 2010, 96, 222504.

(17) Tsujikawa, M.; Oda, T. Finite electric field effects in the large perpendicular magnetic anisotropy surface $\mathrm{Pt} / \mathrm{Fe} / \mathrm{Pt}(001)$ : a firstprinciples study. Phys. Rev. Lett. 2009, 102, 247203.

(18) Velev, J. P.; et al. Magnetic Tunnel Junctions with Ferroelectric Barriers: Prediction of Four Resistance States from First Principles. Nano Lett. 2009, 9, 427-432.

(19) Duan, C. G.; et al. Tailoring magnetic anisotropy at the ferromagnetic/ferroelectric interface. Appl. Phys. Lett. 2008, 92, 122905 .

(20) Mardana, A.; Ducharme, S.; Adenwalla, S. Ferroelectric Control of Magnetic Anisotropy. Nano Lett. 2011, 11, 3862-3867.

(21) Wulfhekel, W.; et al. Single-crystal magnetotunnel junctions. Appl. Phys. Lett. 2001, 78, 509-511.

(22) Liu, M.; Liu, J.; Wang, Q.; Long, S. B.; Zhang, M. H. A metal/ $\mathrm{Al}_{2} \mathrm{O}_{3} / \mathrm{ZrO}_{2} / \mathrm{SiO}_{2} / \mathrm{Si}$ (MAZOS) structure for high-performance nonvolatile memory application. Semicond. Sci. Technol. 2010, 25, 055013.

(23) Qiu, Z. Q.; Pearson, J.; Bader, S. D. Asymmetry of the spin reorientation transition in ultrathin Fe films and wedges grown on Ag(100). Phys. Rev. Lett. 1993, 70, 1006-1009.

(24) Shen, J.; Kirschner, J. Tailoring magnetism in artificially structured materials: the new frontier. Surf. Sci. 2002, 500, 300-322.

(25) Kumar, A.; Fischetti, M. V.; Ning, T. H.; Gusev, E. Hot-carrier charge trapping and trap generation in $\mathrm{HfO}_{2}$ and $\mathrm{Al}_{2} \mathrm{O}_{3}$ field-effect transistors. J. Appl. Phys. 2003, 94, 1728-1737.

(26) Kim, C. J.; et al. Photoinduced memory with hybrid integration of an organic fullerene derivative and an inorganic nanogap-embedded field-effect transistor for low-voltage operation. Adv. Mater. 2011, 23, 3326-3331.

(27) Besset, C.; Bruyere, S.; Blonkowski, S.; Cremer, S.; Vincent, E. MIM capacitance variation under electrical stress. Microelectron. Reliab. 2003, 43, 1237-1240.

(28) Hung, C. C.; et al. An innovative understanding of metalinsulator-metal (MIM)-capacitor degradation under constant-current stress. IEEE Trans. Device Mater. Reliab. 2007, 7, 462-467. 
(29) White, M. H.; Adams, D. A.; Bu, J. K. On the go with SONOS. IEEE Circuits Dev. 2000, 16, 22-31.

(30) Cho, J.; et al. Layer-by-layer assembled charge-trap memory devices with adjustable electronic properties. Nat. Nanotechnol. 2007, 2, 790-795.

(31) Yuasa, S.; Nagahama, T.; Fukushima, A.; Suzuki, Y.; Ando, K. Giant room-temperature magnetoresistance in single-crystal Fe/MgO/ Fe magnetic tunnel junctions. Nat. Mater. 2004, 3, 868-871.

(32) Chiba, D.; et al. Magnetization vector manipulation by electric fields. Nature 2008, 455, 515-518. 